\title{
Retroperitoneal Migration of a Self-Inflicted Ballpoint Pen via the Urethra
}

\author{
Jose Cury, Rafael F. Coelho, Miguel Srougi \\ Division of Urology, School of Medicine, University of Sao Paulo, USP, Sao Paulo, SP, Brazil
}

\begin{abstract}
Numerous accounts documenting the introduction of foreign bodies into the urinary bladder have been reported. These foreign bodies are typically self-inserted via urethral but migration from adjacent organs by an ulcerative process and penetrating injuries are also reported. However, "contrary" migration of a self-inflicted vesical foreign body to the retroperitoneum was not previously reported in literature. We report here a case of a ballpoint pen self-inserted via urethral by a female patient, which was identified in retroperitoneal position years later.
\end{abstract}

Key words: urethra; bladder; foreign-body migration; retroperitoneum; masturbation Int Braz J Urol. 2006; 32: 193-5

\section{INTRODUCTION}

Numerous accounts documenting the introduction of foreign bodies into the genitourinary tract have been reported (1). Most cases are associated with psychiatric disorders, intoxication, and autoerotic stimulation or to get relief of urinary complaints (2). Vesical foreign bodies are typically self-inserted via urethral but migration from adjacent organs by an ulcerative process and penetrating injuries are also reported (1). However, "contrary" migration of a selfinflicted vesical foreign body to the retroperitoneum was not previously reported in literature. We report here a case of a ballpoint pen self-inserted via urethral by a female patient, which was identified in retroperitoneal position years later.

\section{CASE REPORT}

A 38-year-old patient presented to our emergency service with complaint of left iliac fossa pain that had lasted for 12 months and worsened in the last month. The pain was continuous, of light to moderate intensity and was not relieved with common analgesics. The patient also complained of recurrent irritative urinary symptoms, such as dysuria and suprapubic pain, compatible with urinary tract infections. She referred no previous sexual relationships. Physical examination revealed evident pain at palpation of left iliac fossa without peritoneal signs. Genital examination was normal and hymen was not ruptured. Laboratorial findings were normal. A plain abdominal $\mathrm{x}$-ray revealed a radiopaque foreign body in the left iliac fossa compatible with a pen tip (Figure1). Upon further interrogation, the patient referred to autoerotic stimulation with ballpoint pen via the urethra for years. In an undetermined date, the ballpoint pen was lost inside the urethra and migrated probably to the bladder. Under general anesthesia, the patient was submitted to a cystoscopy but no pen, calculus or lesions were found. We opted to perform an exploratory laparotomy through a left Judd-Gibson incision. We founded a rounded fibrous capsule con- 


\section{Retroperitoneal Foreign Body}

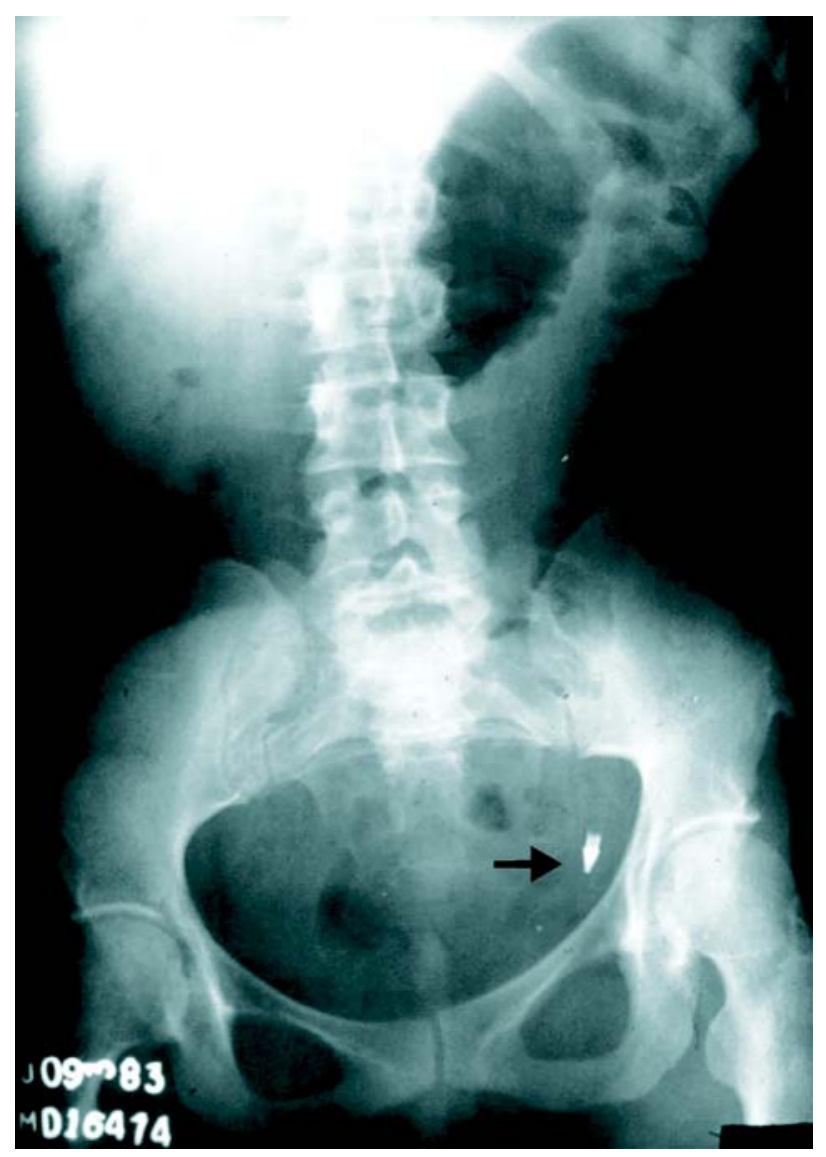

Figure 1 - Abdominal $X$-ray revealed a radiopaque foreign body (white arrow) in left inferior quadrant compatible with a pen tip.

taining the ballpoint pen in the retroperitoneum adjacent to the bladder. The fibrous capsule and the pen were complete excised (Figure-2). Postoperatory was uneventful.

\section{COMMENTS}

Foreign bodies inserted in the urogenital tract represent a urological challenge that often requires prompt intervention (1). The most common reason for such behavior is sexual or erotic in nature, just as in our case patient. Psychiatric disturbances such as borderline personality disorder or schizophrenia may also be present. Patients tend to take time before seeking treatment, usually not before symptoms show up. $\mathrm{X}$-rays are usually enough to diagnose such condi-
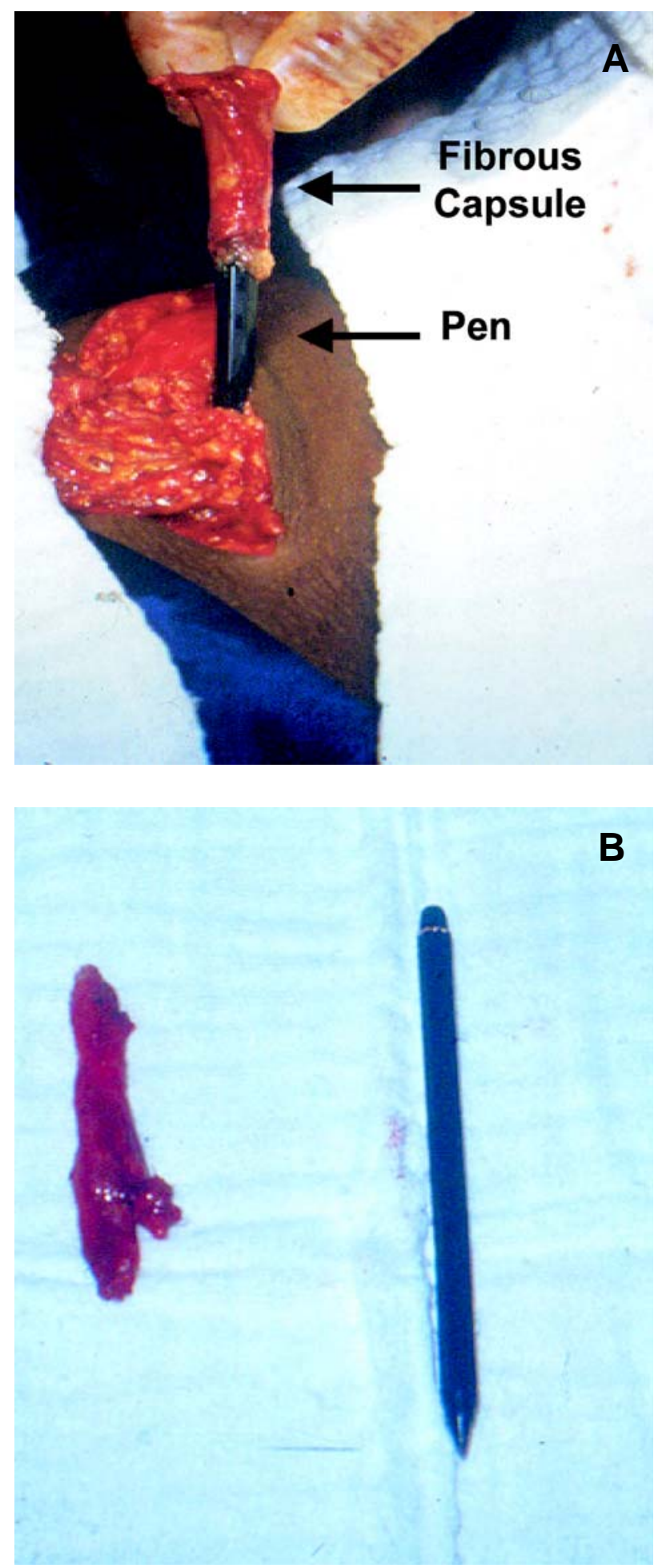

Figure 2 - A) Exteriorization of the pen and fibrous capsule through a left Judd-Gibson incision. B) In detail, the pen and the fibrous capsule excised. 
tions (2). The urethra is the main entrance of foreign bodies in the genitourinary tract and a variety of urethral foreign bodies have been reported in the literature including pencils, electric cable, thermometer, glass rod, toothbrush, candle, serum set, balloon and hairpin among others (1). Vesical foreign bodies are also typically propelled into the bladder and pushed further into the urethra. However, a large number of cases are reported in which various types of foreign bodies migrate into the bladder by an ulcerative process from different organs adjacent to the bladder $(1,2)$. Chicken and fish bones, pins and needles, pencil, thermometer, toothpicks were reported perforating the bladder via gastrointestinal tract. Other foreign bodies that perforate the bladder via vagina are mostly a result of masturbation injuries (1). A case of retroperitoneal foreign body localized after the eroded vaginal wall was also reported (3). However, to our knowledge, this is the first case report of retroperitoneal migration of foreign bodies inflicted via the urethra in the English language. The patient clearly refers to the introduc- tion of the pen in the urethra, not in the vagina, and the integrity of the hymen corroborates this affirmation. Moreover, she refers to recurrent urinary tract infections after the pen introduction. We believe that the pen migrated to the bladder and retroperitoneum through an ulcerative process.

\section{CONFLICT OF INTEREST}

None declared.

\section{REFERENCES}

1. van Ophoven A, deKernion JB: Clinical management of foreign bodies of the genitourinary tract. J Urol. 2000; 164: 274-87.

2. Pal DK, Bag AK: Intravesical wire as foreign body in urinary bladder. Int Braz J Urol. 2005; 31: 472-4.

3. O'Hanlan KA, Westphal LM: First report of a vaginal foreign body perforating into the retroperitoneum. Am J Obstet Gynecol. 1995; 173: 962-4.

Accepted after revision:

February 28, 2006

\author{
Correspondence address: \\ Dr. José Cury \\ Rua Canada, 541 \\ Sao Paulo, SP, 01436-000, Brazil \\ E-mail: jc_uro@osite.com.br
}

